\title{
IMPACTO SOCIAL DE LOS MICROCRÉDITOS: CASO CÁMARA DE COMERCIO DE MILAGRO
}

\author{
Yadira Arteaga Estrella* \\ artestre@hotmail.com \\ yarteagae@unemi.edu.ec
}

\begin{abstract}
RESUMEN
El microcrédito no puede definirse, desde un punto de vista estricto, como herramienta de desarrollo utilizada exclusivamente en los países pobres. En la actualidad, esta herramienta es válida en distintos entornos y diferentes condiciones. Para gestionar los microcréditos hay que considerar varios factores: los que influyen en su eficacia medida en términos de ganancia, de bienestar del beneficiario y aquellos que influyen en su eficiencia, es decir, en el rendimiento del microcrédito. De ahí la necesidad de realizar la investigación formativa; en la que se da la interrelación docentes - estudiantes cuyo objetivo es interpretar el uso de los microcréditos obtenidos para el desarrollo de las actividades productivas de los socios de la Cámara de Comercio en la ciudad de Milagro cuyo fundamento es mejorar la cultura investigativa e incrementar el interés que tenemos los docentes en relación a la investigación en nuevos procesos, en búsqueda de nuevos conocimientos que propicien la creatividad e innovación de nuevas ideas. La finalidad de la estrategia es desarrollar habilidades y transferencia del aprendizaje a través de la sistematización de nuevos conocimientos la misma se aprende fundamentalmente a través del trabajo en el campo de acción.
\end{abstract}

Palabras clave: impacto social, microcréditos, Cámara de Comercio.

\begin{abstract}
Microcredit can not be defined from a strict point of view, as a development tool used exclusively in poor countries. Currently, this tool is valid in different environments and different conditions. To manage microcredit must consider several factors that influence the effectiveness measured in terms of profit, welfare beneffic those that influence their efficiency, ie the performance of microcredit. Hence the need for formative research which gives the relationship teachers - students whose goal is to interpret the use of microcredit obtained, for the development of the productive activities of the members of the Chamber of Commerce in the city whose foundation Miracle is to improve the research culture and increase the interest we have teachers in relation to research on new processes, in search of new knowledge, foster creativity and innovation of new ideas. the purpose of the strategy is to develop skills and learning transfer through the systematization of new knowledge it is learned primarily through work in the field.
\end{abstract}

Keywords: social impact, microcredit, Cámara de Comercio.

\footnotetext{
* Máster en Diseño Curricular, Economista, Diplomada en Diseño Curricular por Competencias, Docente de la Unidad Académica Ciencias Administrativas y Comerciales de la Universidad Estatal de Milagro.
} 


\section{INTRODUCCIÓN}

La finalidad del microcrédito es proporcionar al usuario un crédito pequeño para realizar una actividad económica que le permita independencia. En 1976, Yunus fundó el Banco de Grameem en Bangladesh (India), el microcrédito está presente en muchos países, incluyendo los países ricos, los cuales empiezan a utilizar como medida de desarrollo económico para los menos desfavorecidos [1].

El microcrédito no puede definirse desde un punto de vista estricto cuya utilización se dé sólo en los países pobres. En la actualidad, es válida en distintos entornos y diferentes condiciones. Para gestionar los microcréditos hay que considerar varios factores: Los que influyen en su eficacia medida en términos de ganancia, de bienestar del beneficiario, y aquellos que influyen en su eficiencia, es decir en el rendimiento del microcrédito o en la relación entre los recursos económicos prestados y los recursos o beneficios obtenidos, el principal objetivo de los microcréditos es la lucha contra la pobreza y la desigualdad económica social.

La investigación formativa tiene como fundamento mejorar la cultura investigativa e incrementar el interés que tenemos los docentes en relación a la investigación en nuevos procesos, en búsqueda de nuevo conocimiento, que propicien la creatividad e innovación de nuevas ideas. La finalidad de la estrategia es desarrollar habilidades y transferencia del aprendizaje a través de la sistematización de nuevos conocimientos, la misma que se aprende fundamentalmente a través del trabajo en el campo de acción, con la supervisión de un docente que le ayuda a construir a través de sus experiencias.

Los atributos del docente tienen que ver con el conocimiento y manejo del método científico, ya que el aprendizaje por descubrimiento requiere que el profesor sea respetuoso de las posiciones de los estudiantes, sobre todo de las posiciones divergentes, que tenga capacidad de conducción de los grupos, de su actualización permanente y de su reflexión constante en su práctica pedagógica, le permiten construir ensayando alternativas y validándolas para mejorar sus práctica, las que aportan al aprendizaje de sus estudiantes para "formarlos" en investigadores a través de su práctica, sin necesidad de un proyecto concreto de investigación [2].
De ahí la necesidad de realizar la investigación formativa en la que se da la interrelación docentesestudiantes cuyo objetivo es interpretar el uso de los microcréditos obtenidos, para el desarrollo de las actividades productivas de los socios de la Cámara de Comercio en la Ciudad de Milagro.

\section{FUNDAMENTACIÓN}

En Ecuador, el sistema financiero lo constituyen 22 bancos privados, 3 instituciones financieras Públicas, 28 cooperativas de ahorro y crédito, que se encuentran reguladas por la Superintendencia de Bancos y la Dirección Nacional de Cooperativas que se encargan del control de aproximadamente 311 cooperativas de ahorro y crédito. Existiendo otras instituciones dedicadas a financiar pequeños proyectos que no están consideradas dentro del sistema financiero, cajas de ahorro, que son organizaciones que pueden estar integradas por personas que pertenecen a una institución [3].

Los microcréditos pueden ayudar a dar préstamos a clientes de éxito y los que no son sujetos de crédito logren ir a sistemas informales a pedir un préstamo, lo que experimenta un impacto dentro de la evolución de su negocio.

\section{El impacto en los servicios financieros}

Para entender a la pobreza en todos sus aspectos, es importante moverse de lo económico para incluir la satisfacción de necesidades básicas (alimentos, vivienda, vestido, salud, educación y bienestar psicológico), los medios para lograr bienestar en el presente y futuro, capital social y capacitación, y vulnerabilidad ante el riesgo.

Con el objeto de entender mejor el papel del microcrédito y su función en la reducción de la pobreza, es importante entender conceptualmente los mecanismos por los que los servicios financieros pueden afectar la vida de la gente que menos tiene [4].

La gente vive en un entorno cambiante y de alto riesgo. Necesitan ser capaces de aprovechar las oportunidades que conducen a un mejoramiento del ingreso y del estatus económico para protegerse a sí mismos en contra de los riesgos de la crisis o eventos repentinos y enfrentarlos cuando estos se presentan. La reducción de la pobreza, es entonces una parte del proceso del incremento de ingreso y estabilidad económica, lo cual conduce a una mejor satisfacción de las necesidades, básicas y acceso a los servicios [5]. 
Entender por qué la gente necesita el dinero, cómo los servicios financieros pueden ser útiles y qué impactos (positivos y negativos) puede tener en la vida de las personas.

Existen cuatro áreas que se consideran importantes para obtener un crédito:

1. Oportunidades: La gente necesita dinero con el objeto de invertir en oportunidades económicas o sociales. El dinero puede invertirse como capital de trabajo o como bienes de producción para una actividad remunerativa. Otras oportunidades podrían no estar relacionadas con el retorno de la inversión.

2. Consumo: frecuentemente hay una diferencia entre los patrones de consumo de la gente y su ingreso. Los patrones de consumo familiar, compras regulares o grandes compras ocasionales, para ropa, compra de comida y despensa o artículos del hogar; el ingreso puede ser uniformemente distribuido a través del año o puede ser errático. En ambos casos, el dinero podría no estar disponible cuando sea necesario y conducir a una administración ineficiente de las finanzas familiares.

3. Necesidades del ciclo de vida: Toda la gente tiene grandes sucesos predecibles a lo largo de sus vidas, por ejemplo: nacimiento, enfermedad, matrimonio, muerte, pagos escolares, jubilación, entre otras. Todos estos requieren relativamente grandes sumas de dinero.

4. Emergencias y crisis: Eventos impredecibles, tales como enfermedad, muerte, accidente, incendios, clima y crimen son comunes para toda la gente y más comunes para los muy pobres, quienes son también más vulnerables a sus impactos negativos [6].

\section{METODOLOGÍA}

La investigación formativa interrelaciona docentes-estudiantes para propiciar mejorar sus habilidades cognoscitivas:

Analítica, crítica, pensamiento productivo, que den solución a los problemas que se plantean, construyendo en los docentes la cultura de evaluación permanente de su práctica a través de procesos investigativos. De ahí nace la necesidad del cambio permanente en nuestras prácticas de investigación formativa [7].
La investigación con enfoque de estudio de casos permite generalizar lo que se está estudiando a profundidad, en relación a la Cámara de Comercio de Milagro. El investigador goza del privilegio de aseverar lo que considere significativo como resultado de lo investigado. En sus informes, aparecerán los hallazgos percibidos y la responsabilidad de la interpretación [8].

El presente trabajo de investigación formativa fue dado con los estudiantes de cuarto semestre de Economía de la Universidad Estatal de Milagro en el año 2011. La población de estudio fue la Cámara de Comercio de Milagro y se enmarca en un enfoque cualitativo, considerando que este está en relación directa con la naturaleza del problema u objeto del estudio. Dentro del enfoque cualitativo, optamos como metodología de la investigación, por un estudio de casos múltiple, dado que la muestra del estudio está constituida por los Socios de la Cámara de Comercio de Milagro que fueron las fuentes de información y de la aplicación de varios instrumentos de recolección de datos.

Ellos nos lleva a ensayar métodos prácticos, es decir, es la que nos introduce en el campo de la práctica y a partir de allí se va logrando cierta efectividad.

Es necesario en esta modalidad tener en cuenta los siguientes pasos: reflexión sobre la propia práctica, práctica mediante observación, estructura de la práctica y construcción de prácticas mejoradas; al culminar el trabajo de campo que nos permita realizar investigación para cuestionarlos y mejorarlo para construir una adecuada investigación [9].

Con respecto al proceso de análisis de los datos, recorremos al enfoque mixto de análisis, es decir, deductivo-inductivo [10].

INTEGRACIÓN DE LA TEORÍA Y LA PRÁCTICA. La importancia de la transformación de la teoría con la práctica, la sistematización de nuevos conocimientos, validando a través de una consciente crítica, dado por los datos del diario de campo aportados por técnicas de observación [11].

\section{PROBLEMA}

Escaso conocimiento del uso del microcréditos en las actividades productivas de los socios de la Cámara de Comercio en la ciudad de Milagro 


\section{POBLACIÓN}

Es el conjunto de elementos en el que se da referencia sobre las observaciones que se realizan [12].

\begin{tabular}{|l|l|l|l|}
\hline MICROEMPRESARIO & COMPAÑÍAS & MUJERES & HOMBRES \\
\hline 692 & 57 & & \\
\hline TOTAL 635 & & 162 & 473 \\
\hline
\end{tabular}

Cuadro N. ${ }^{1}$ : Información Cámara de Comercio de Milagro

\section{POBLACIÓN OBJETO DE ESTUDIO}

\begin{tabular}{|l|l|l|}
\hline MICROEMPRESARIO & MUJERES & HOMBRES \\
\hline TOTAL 635 & 162 & 473 \\
\hline
\end{tabular}

Cuadro N. ${ }^{\circ}$ 2: Información Cámara de Comercio de Milagro

\section{POBLACIÓN FINITA}

Es el número de elementos conocidos. Que la forman es finito, ya que conocemos la población objeto de estudio 635 Socios de la Cámara de Comercio de Milagro [13].

FÓRMULA POBLACIÓN FINITA

$$
\begin{aligned}
& \mathbf{n}=\frac{N}{(E)^{\mathbf{2}(N-\mathbf{1})+\mathbf{1}}} \\
& \mathrm{n}=\frac{635}{(0,05) 2(635-1)+1} \\
& \mathrm{n}=\frac{635}{(0.0025)(634)+1} \\
& \mathrm{n}=\frac{635}{(1.585)+1} \\
& \mathrm{n}=\frac{635}{2.585} \\
& \mathbf{n}=\mathbf{2 4 5 , 6 4 7}
\end{aligned}
$$

\section{MUESTREO ALEATORIO SIMPLE}

La forma más común de obtener una muestra es la selección al azar. Es decir, cada uno de los socios de la población objeto de estudio Cámara de Comercio de Milagro, tienen la misma posibilidad de ser elegido. Si no se cumple este requisito, se dice que la muestra es viciada. Para tener la seguridad de que la muestra aleatoria no es viciada, debe emplearse para su constitución una tabla de números aleatorios [14].

\section{MUESTRA}

Es un subconjunto de unidades de análisis de una población dada, destinado a suministrar información sobre la población. Para que este subconjunto de unidades de análisis sea de utilidad estadística, la muestra es de 245 personas, deben reunirse ciertos requisitos en la selección de los elementos [15].

Para la obtención de la información del presente proyecto, se realizaron varias técnicas, las 
cuales nos permitirá tener una visión clara del problema, por el cual están atravesando los socios de la Cámara de Comercio [16].

\section{TÉCNICA}

\section{Encuesta}

Es la técnica que a través de un cuestionario adecuado nos permite recopilar datos de la muestra de 245 socios, la misma que la obtuvimos de la fórmula de población finita. En la investigación, los estudiantes realizaron las encuestas, y que fueron supervisadas por el docente de forma clara, sencilla, y precisa.

\section{Entrevista}

La entrevista es una conversación entre dos o más personas, en la cual uno es el que pregunta (entrevistador) y el otro el entrevistado [17]. Los estudiantes dialogaron con los socios con el objetivo de interpretar el Impacto de los microcréditos obtenidos, en el desarrollo de sus actividades productivas.

\section{RESULTADOS}

El crédito es una deuda que conlleva entonces el potencial de conducir a diferentes impactos.

Hay muchos problemas de quienes empezaron un negocio con dinero de un crédito en ocasiones, negocios fallidos, que dejo como resultado endeudamiento, creciente vulnerabilidad, tensión en el hogar y experiencias negativas y con una deuda que no pudieron pagar, muchos pasaron este problema serrando sus negocios, otros cambiando de negocios y prestando más dinero para empezar, sin tener la capacitación o la experiencia de iniciar un negocio [18].

La investigación formativa del caso de la Cámara de comercio de milagro, nos permitió ANALIZAR que quienes tuvieron acceso a un crédito, lograron mejorar e incrementar las ganancias en su microempresa [19].

\begin{tabular}{|l|c|c|c|c|c|}
\hline & Privado & Público & Otros & Ninguno & Total \\
\hline $\begin{array}{l}\text { ¿Ha recibido financiamiento de una } \\
\text { entidad financiera? }\end{array}$ & $35 \%$ & $27 \%$ & $18 \%$ & $20 \%$ & $100 \%$ \\
\hline & & & & & \\
\hline
\end{tabular}

FUENTE: SOCIOS DE LA CÁMARA DE COMERCIO DE MILAGRO

Cuadro N. ${ }^{\circ}$ 3. Pregunta: ¿Ha recibido financiamiento de una entidad financiera?

\section{HA RECIBIDO FINANCIAMIENTO DE UNA INSTITUCIÓN FINANCIERA}

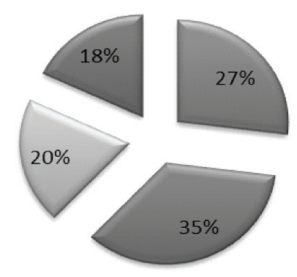

๑PÚBLICO $\square$ PRIVADO $\triangle N I N G U N O$ 口OTROS

Gráfico N. ${ }^{\circ} 1$ Resultados de Investigación formativa, muestra de 245 socios de la Cámara de Comercio de Milagro.

En cuanto al financiamiento de los distintos negocios, podemos decir que el $35 \%$ de las microempresas están conformadas por capital de instituciones financieras privadas, y el $27 \%$ escogieron capitalizarse por una institución pública como lo son el Banco Nacional de Fomento, Corporación Financiera Nacional, entre otras.
El $18 \%$, han realizado crédito al sector no financiero, sea este determinado por familiares que viven en el exterior o quienes prestaron dinero a terceras personas sin interés referenciado por el Banco Central del Ecuador. Otro mecanismo es utilizar créditos a través de terceras personas, debido a que se encuentran en central de riesgo y no cuentan con un buen historial crediticio.

El grupo ninguno hace referencia al $20 \%$ de socios que tienen su negocio por más de 5 años y han logrado tener estabilidad y se capitalizan de sus ganancias en sus negocios.

Los microcréditos conducen a la estabilización del ingreso y a la suavización del gasto: Las familias del nivel bajo y medio se ven impactadas por el préstamo sobre el ingreso familiar y tienen excelentes oportunidades para inversión, así como a acceder a mejores condiciones de mejorar sus niveles de supervivencia, afirmando que para usar un crédito de manera eficaz, las familias necesitan haber alcanzado previamente un "nivel económico mínimo" [20] . 
Tal es el caso del señor Kleber González, quien en sus inicios obtuvo un crédito con un banco privado e inició la compra venta de arroz. En la actualidad ha logrado mejorar su calidad de vida e invertir en otra línea en su negocio.

\section{Trabajar para pagar el crédito}

El endeudamiento del microempresario es uno de los desafíos que debe ser mejorado, potencializarse para mejorar su situación socio-económica. Algunas veces el microcrédito lo empeora por crear deudas a corto tiempo y altas tasas de interés que pueden hacer difícil pagar el crédito. En algunas ocasiones, el microempresario no sabe cómo usar el crédito de manera efectiva y lograr los resultados esperados. Es necesario asesorar al microempresario antes de tomar el crédito y brindar capacitación en plan del negocio, administración, entre otras.

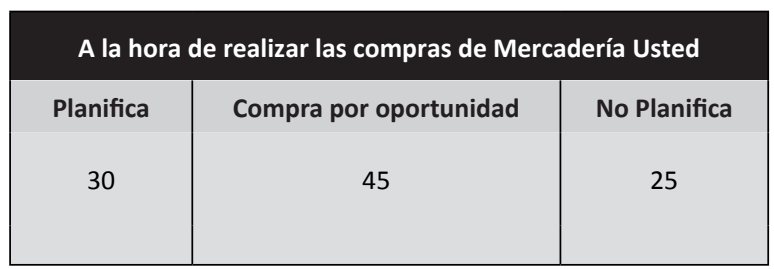

FUENTE: SOCIOS DE LA CÁMARA DE COMERCIO DE MILAGRO

Cuadro N. ${ }^{\circ} 4$ Pregunta: $¿$ A la hora de realizar las compras de mercadería usted?

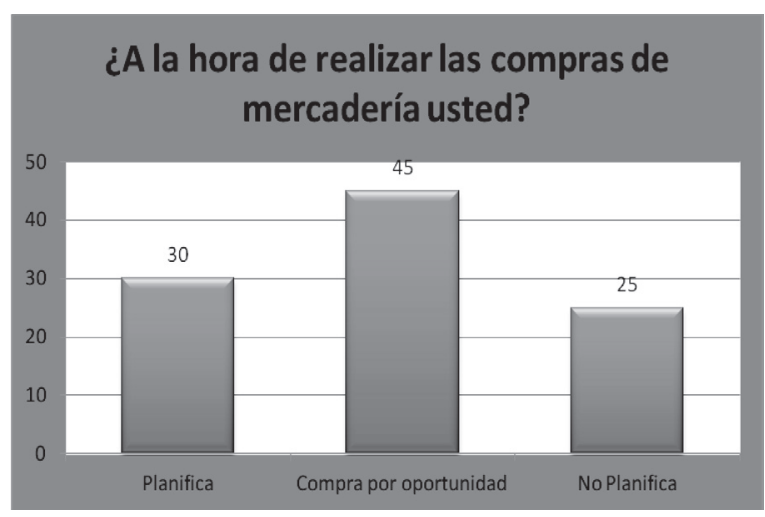

Gráfico N. ${ }^{\circ} 4$ Resultados de Investigación formativa, muestra de 245 socios de la Cámara de Comercio de Milagro

Al momento de comprar la mercadería, pudimos descubrir que el 30\% de los socios entrevistados planifican para hacer algún pedido, mientras que el $45 \%$ no supieron contestar como adquieren la mercadería para obtener oportunidad en el precio.

Por otro lado, vemos que el $25 \%$ no planifica sino que analizan las tendencias y la preferencia que tienen los clientes para realizar las compras de mercadería en su negocio.

Ahora solo estamos sobreviviendo.

\begin{tabular}{|l|c|c|c|c|}
\hline & Administrativo & Financiero & Legales & Total \\
\hline $\begin{array}{l}\text { ¿Cuáles } \\
\text { han sido los } \\
\text { problemas en } \\
\text { el manejo de } \\
\text { su negocio? }\end{array}$ & $25 \%$ & $45 \%$ & $30 \%$ & $100 \%$ \\
\hline
\end{tabular}

FUENTE: SOCIOS DE LA CÁMARA DE COMERCIO DE MILAGRO

Cuadro N. 5 Pregunta: ¿Cuáles han sido los problemas en el manejo de su negocio?

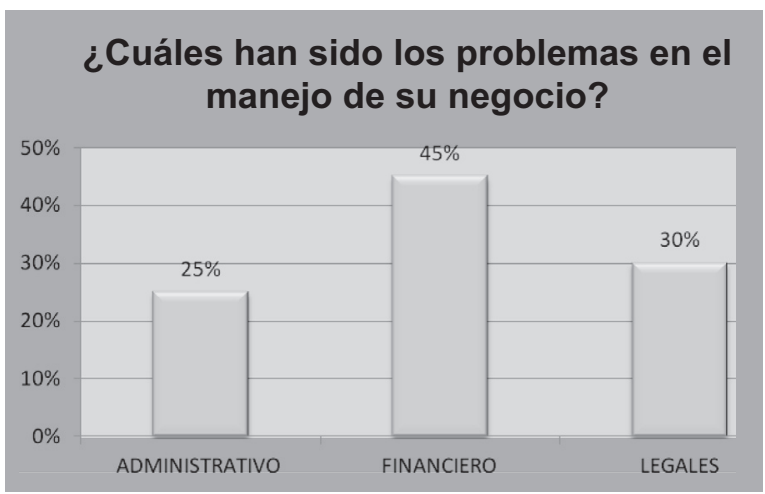

Gráfico N. ${ }^{\circ} 5$ Resultados de Investigación formativa, muestra de 245 socios de la Cámara de Comercio de Milagro

Para conocer qué tipo de problemas han enfrentado los empresarios en el trascurso que lleva ejerciendo su actividad económica, las dividimos en tres: administrativas, financieras y legales.

Podemos decir que uno de los problemas comunes que enfrentan las personas al momento de tener su negocio son los de tipos financieros, seguidos por los legales y administrativos.

De nuestra muestra, el $45 \%$ ha tenido problemas de carácter financiero, seguido por los problemas legales con el 30\% y administrativos 25\%

(Zaida Neira) inicio con dinero de un préstamo familiar, su capital se vio incrementado, actualmente tiene un negocio de ropa; es una microempresaria y ha tenido que competir con ropa barata de distintos países: Perú, China, Colombia que ha invadido el mercado milagreño. El temor de realizar un crédito no le permite mejorar e incrementar sus ingresos. Otro problema es que la producción textil se ha visto disminuida, así menciona la costurera, señora Guadalupe: el trabajo se ha reducido, tiene tengo escasas ganancias; solo lo suficiente para poder sobrevivir, aun después de interminables horas de trabajo. 
Para las costureras con más de veinte o treinta años de experiencia, no hay mejores opciones para ser rentable, ya que no saben hacer otra cosa mejor. Los créditos los toman no para comprar tecnología, contratar a más gente o desarrollar su negocio, lo toman para comprar materias primas, telas nuevas que le permitan adaptarse a las nuevas condiciones del mercado a través de innovación. Así, ellos introducen nuevos productos y buscan a nuevos clientes y logran mantenerse a pesar de todos los desafíos.

La necesidad por mantenerse está lejana de las ganas de crecer y por eso pocos tienen perspectivas futuras para sus negocios: van adaptándose, o en sus palabras, van luchando.

El microcrédito ayuda a los microempresarios a solucionar problemas. Obtener mucho dinero, es un riesgo grande, no es fácil operar con cuotas grandes y con un corto plazo [21].

Se deben utilizar mecanismos de flexibilización para tomar los préstamos con flexibilidad y que respondan a problemas particulares del sector microempresarial. Meditar que el pequeño microempresario no es una réplica del gran empresario. Considerar al microempresario y sus características culturales, económicas y sociales distintas de los demás [22].

Los microcréditos conducen a un incremento en la acumulación de bienes.

Un impacto clave del microcrédito es ayudar al cliente a acumular o retener bienes físicos. Los bienes crecen, ya sea a través del uso directo de préstamos, como un beneficio de la suavización del ingreso, o a través del uso de ganancias generadas por medio de la inversión del préstamo [23].

En el caso del $(+)$ Sr. Roberto Santur, producto de su buena categorización del crédito en el sistema financiero, obtuvo varios préstamos, y logro así:

1) Bienes familiares que primordialmente contribuyeron a mejorar su calidad de vida y proveer seguridad de los ingresos en el caso de una futura necesidad.

2) Bienes familiares que son primordialmente mantenidos como ahorro en especie.

3) Bienes productivos que son usados para generar ingresos en el equipamiento de los negocios.

\section{IMPACTO DE GÉNERO}

Una de las motivaciones para enfocarse en las mujeres en lugar de los hombres, es la evidencia de las mejoras económicas experimentadas y controladas por mujeres que conducen a optimizar el bienestar de la familia entera, particularmente cuando van acompañadas por un incremento en el empoderamiento de las mujeres.

Caso Señora Abarca. El crédito permite a las familias mejorar su capital humano, lo cual a su vez permite mejorar y elevar el estándar de vida en el presente y proyectarse hacia el futuro [24].

La motivación de dirigirse a las mujeres es frecuentemente instrumental, basada en que las mujeres son más cumplidas, confiables y fáciles de encontrar, hay también importantes motivaciones, crecimiento económico, mejorar la educación para los hijos, independiente de la manera en la que los créditos hayan sido entregados [25].

El impacto positivo en la mujer al obtener un crédito, le permite mejorar su situación económica y tener mayor participación dentro de la sociedad, con responsabilidad y poder de decisión en la economía familiar demostrando resultados más beneficiosos [26].

La capacidad económica de las mujeres incrementa los recursos familiares necesarios para mejorar la seguridad alimentaria, salud y nutrición del hogar e incrementar la capacidad económica de las mujeres...Los cambios económicos y del bienestar tienen impacto en las relaciones sociales y en la confianza y bienestar de los clientes [27].

Los cambios económicos pueden conducir a que las mujeres participen más ampliamente en actividades comunitarias o fuera de la comunidad y su aporte dentro de la sociedad [28].

\section{Impacto de los oficiales de crédito}

El personal que labora como oficial de crédito debe incentivar sin excluir a los más pobres. Ya que ellos cuentan con más problemas para acceder a un microcrédito en una institución financiera [29].

Los oficiales de crédito deben sujetarse a las políticas de la institución, manteniendo sus niveles de productividad, e incrementar su cartera de préstamos de manera excepcional y alcanzar un mayor número de clientes medido desde resultados, sin verificación del uso y beneficio del préstamo y el impacto que pudo generar dentro del grupo más vulnerable, gente que no es dueña 
de un negocio iEl mito de la gente económicamente activa! [30].

El crédito invertido como capital de trabajo o como bienes de producción en una empresa remunerativa conduce al establecimiento de una empresa nueva o al crecimiento de una ya existente. Las utilidades de la empresa proveen un ingreso creciente y un fortalecimiento general de las fuentes de ingreso [31].

Es importante distinguir las necesidades de los clientes entendiendo su mercado. Asegurándose que están ofreciendo los productos adecuados para los clientes que desean servir. Uno de los problemas es que no hay una planificación de las necesidades reales de su negocio.

\begin{tabular}{|l|c|c|c|c|}
\hline & $\begin{array}{c}\text { Considero } \\
\text { importante }\end{array}$ & $\begin{array}{c}\text { Lo he } \\
\text { pensado }\end{array}$ & $\begin{array}{c}\text { Me es } \\
\text { indiferente }\end{array}$ & Total \\
\hline $\begin{array}{l}\text { ¿Considera } \\
\text { usted que la } \\
\text { capacitación } \\
\begin{array}{l}\text { le permite } \\
\text { mejorar el } \\
\text { manejo de } \\
\text { su negocio? }\end{array}\end{array}$ & $51 \%$ & $31 \%$ & $18 \%$ & $100 \%$ \\
\hline
\end{tabular}

Cuadro N. ${ }^{\circ} 6$ Pregunta: ¿Considera usted que la capacitación le permite mejorar el manejo de su negocio?

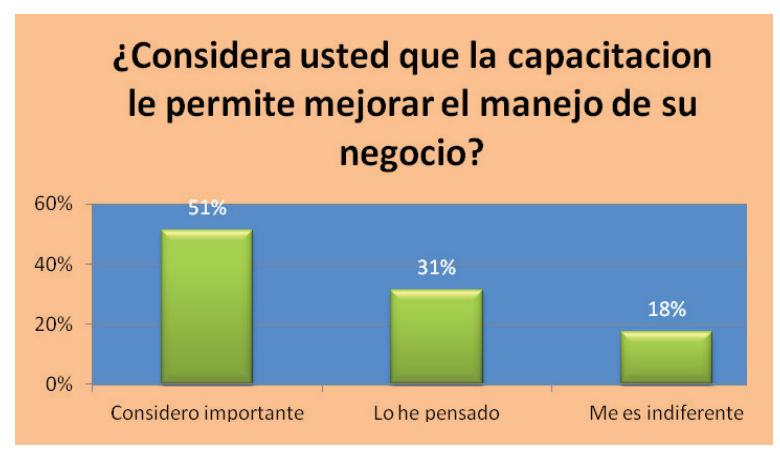

Gráfico N. ${ }^{\circ} 6$ Resultados de Investigación formativa, muestra de 245 socios de la Cámara de Comercio de Milagro

El 51\% de los microempresarios están de acuerdo en que la capacitación continua aporta el mejoramiento del negocio, por lo cual consideran un elemento importante para el crecimiento de su economía; otro $31 \%$ simplemente piensa que tomar seminarios aportaría en el mejor manejo del negocio. El 18\% considera que la capacitación no es importante para el crecimiento de su negocio.

\section{El problema de la no medición del impacto}

La evaluación del impacto es la de medir los resultados de una inversión y mejorar la práctica de una institución de microcréditos. Las herramientas para medir el impacto que se concentran en mejorar, en vez de comprobar, pueden incorporar algunas de las herramientas que ya se usan, pero las utilizarán de una manera diferente. Las herramientas que usan los administradores en forma periódica adoptarán un enfoque distinto [32].

Las instituciones financieras no están respondiendo si el dinero dado en los microcréditos está logrando mejorar la vida de aquellas personas que no tienen recursos. No prestan asistencia alguna en la evaluación de los distintos tipos de sistemas de microcréditos. Las instituciones que otorgan microcréditos son entes rentables y atienden a la misma cantidad de clientes, el impacto de una institución a otra difiere. Aun proporcionando créditos financieramente sostenibles, tienen un impacto marginal y aislado en sus clientes [33].

Una institución financiera (cooperativa) puede formar líderes de redes sociales entre sus clientes, de manera tal que produce un amplio cambio positivo en las comunidades. El objetivo de la institución financiera, no solo debería medir financieramente sus resultados, sino participar activamente en la evaluación del impacto para medir la gestión de los microcréditos.

Es importante realizar un seguimiento desde cuando el cliente ingresa al programa indicando si el impacto aumenta o disminuye con el correr del tiempo, analizando los cambios de los clientes nuevos con los potenciales. Contratar personas especialmente capacitadas para realizar las investigaciones o interpretar sus resultados, que sirva para medir bien el impacto en forma periódica. Considerar el estudio del impacto del donante, cuyo objetivo es comprobar si los programas financiados tuvieron algún resultado positivo.

\section{CONCLUSIONES}

1. Podemos evidenciar que el capital de trabajo de los microempresarios proviene de entidades financieras privadas en un 55\%, el 27\% escogió capitalizarse por una institución pública, como lo son el Banco Nacional de Fomento, Corporación Financiera Nacional, entre otras. 
2. El $18 \%$ dio como resultado socios que han obtenido créditos en el sector no financiero, sea este determinado por familiares que están en el exterior que le prestaron el dinero, otros optaron por obtener préstamos con interés altos, o créditos obtenidos por terceras personas, debido a encontrarse en central de riesgo.

3. Los socios que tienen un negocio por más de 5 años han logrado tener estabilidad y se capitalizan producto de las ganancias de sus negocios.

4. Al momento de comprar la mercadería pudimos descubrir que el $30 \%$ de los socios entrevistados hacen una planificación para hacer algún pedido, mientras que el $45 \%$ nos supieron contestar como adquieren la mercadería para obtener alguna oportunidad en el precio.

5. Por otro lado, vemos que el $25 \%$ realiza una investigación previa del mercado para ver la tendencia y la preferencia que tienen los clientes para así adquirir la mercadería y evitar que se estanque en el negocio.

6. El 51\% de los microempresarios están de acuerdo en que la capacitación continua aporta el mejoramiento del negocio, por lo cual consideran un elemento importante para el crecimiento de su economía, otro $31 \%$ simplemente piensa que tomar seminarios aportaría en el mejor manejo del negocio. El 18\% consideran que la capacitación no es importante para el crecimiento de su negocio.

7. Se pudo analizar que uno de los problemas comunes que enfrentan los socios de la Cámara de Comercio de Milagro en su negocio son financieros con un $45 \%$, legales con un $30 \%$ y administrativos un $25 \%$.

\section{REFERENCIAS BIBLIOGRÁFICAS}

[1]GUERRA, ÁNGEL. El banquero de los pobres. (2005). España: Editorial Paidos. Consultado en: GrameenCommunicationshttp://www. grameen-info.org

[2] VILLAVECES, J. L. (2001). Los grupos de investigación. En: educación superior, desafío global y respuesta nacional. Colombia: Alfomega.

[3] Banco Central del Ecuador. Cooperativismo en el Ecuador. pág.56
[4]RUTHERFORD, STUART. (1999). The Poor and Their Money. (New Delhi, India: Oxford University Press, 1999); Graham Wright, Examining the Impact of Microfinance Services. Increasing Income or Reducing Poverty Small Enterprise Development 10, no. 1 (1999); Sebstad and Cohen, Microfinance

[5]ROBINSON, MARGUERITE, S. (2001). The Microfinance Revolution: Sustainable Finance for the Poor. Washington, DC: The World Bank.

[6]SEBSTAD AND COHEN, en Microfinance 33; describe el riesgo de la siguiente manera: la probabilidad de una pérdida o la pérdida misma; el riesgo tiene muchas fuentes. 1. Factores estructurales como la temporalidad, la inflación o el clima; 2 . Crisis y emergencias no anticipadas, como la enfermedad o muerte de un familiar, la pérdida del empleo, incendios y robos, y 3 . Los altos costos asociados con eventos del ciclo de vida, como matrimonios, funerales y la educación de los hijos. Igualmente, los riesgos están asociados con 4. La operación de una empresa y 5. La toma de un préstamo.

[7]RESTREPO, B. (1999). Escuela Investigadora e Investigación de Aula. Cuadernos pedagógicos N. ${ }^{\circ}$ 14, Universidad de Antioquia: Medellín.

[8]GARCÍA-VALCÁRCEL, A. (2001). La formación docente del profesor universitario, su formación y desarrollo profesional. Didáctica Universitaria. Madrid, La Muralla, 9-43.

[9]FERNÁNDEZ, A. (2004). El portafolio como estrategia formativa y de desarrollo profesional. Educar, 33, 127-142.

[10]STENHOUSE, L. (1.993). La investigación como base de la enseñanza, Madrid, Morata 11.

[11](CRESWELL, 1998; MERRIAM, 1998; STAKE, 1995).

[12]RUIZ MUÑOZ, DAVID. (2004). Manual de Estadística. España: editorial Española.

[13]HERNANDEZ SAMPIERI, ROBERTO. (2005). Metodología de la investigación. México: Ediciones Castillo.

[14]SURENDRA P., VERMA. (2006). Estadística Básica para el manejo experimental. Universidad Nacional Autónoma de México. 
[15]SARABIA SÁNCHEZ, F. J. (1999). Metodología para la investigación en marketing y dirección de empresas. Madrid, Pirámide.

[16]RIALP I, CRIADO J. (2003). Escalas de Medición y Temas relacionados, Departamento de Economía de la Empresa, Facultad de Económicas, Universidad Autónoma de Barcelona.

[17]SEBSTAD AND COHEN, Microfinance; Snodgrass and Sebstad, Clients; Wright, Examining the Impact.

[18]NEWBOLD, P. (1996). Estadística para los negocios y la economía. Editorial Prentice-Hall.

[19]SURENDRA P.VERMA. Op. cít., p. x.

[20]SARABIA SÁNCHEZ, F. J. Op. cít., p. x.

[21]RIALP I., CRIADO J. Op. cít., p. x.

[22]SEBSTAD AND COHEN, Microfinance; Snodgrass and Sebstad, Clients; Wright, Examining the Impact.

[23]CALDERÓN, Jorge. (2007). Reflexiones sobre los límites y alcances en la formación de investigadores. Revista, Uni-pluri/versidad. Vol. 7. N. ${ }^{\circ}$, pp. 14-22.

[24]CERDA, Gutiérrez, Hugo (2007). La investigación formativa en el aula. La pedagogía como investigación. Bogotá: Cooperativa Editorial Magisterio.

[25]Comisión Económica para América Latina y el Caribe - CEPAL. Seminario Internacional las diferentes expresiones de la vulnerabilidad Social en América Latina y el Caribe. Santiago de Chile, 20 y 21 de junio de 2001.
[26]GARNICA MONCAYO, Christian Paúl. Diseño de un modelo de mejoramiento en los procesos de una microempresa de servicios de gestión Bancaria. Consultado en: http://bibdigital.epn.edu.ec/bitstream/15000/2093/1/ CD-0997.pdf

[27]SEBSTAD Y COHEN. Examinando el Impacto de las Micro finanzas.

[28]BOLETÍN INFORMATIVO DE BANCO FINCA. Consultado en: http://www.bancofinca.com/ verTestimonios.aspx?idC $=1 \&$ idNot $=10$

[29]SIMANOWITZ, Anton, WALTER, Alicia. Impacto Seguro, Como Servir al más pobre construyendo Instituciones financieramente autosuficientes Consultado en: http://www.microcreditsummit.org/papers/ sp_simanowitz-ei.pdf

[30]GONZÁLEZ, Sebastián, LÓPEZ Pascual, ALTINA, JOAQUIN. (2001). Gestión bancaria: los nuevos retos en un entorno global. Editorial McGraw-Hill.

[31]SANCHIS PALACIO, J. CAMPS TORRES, Joaquín. (2003). Administración Bancaria. País: Editorial Ediciones Díaz de Santos.

[32]JESÚS HUERTA DE SOTO. Dinero, Crédito Bancario y Ciclos Económicos. Editorial Unión Editorial S.A.

[33]GARRIDO RICCO, Silvia. Evaluación de Impacto. Microcrédito Social. Observatorio de la Inclusión Social. Universidad Autónoma de Madrid. 